\title{
Respiratory Rate Detection by a Time-Based Measurement System
}

\author{
E. Sifuentes, J. Cota-Ruiz, R. González-Landaeta
}

Departamento de Ingeniería Eléctrica y Computación, Universidad Autónoma de Ciudad Juárez, ChihuahuaMéxico.

\section{ABSTRACT}

This paper proposes a system that converts a time-modulated signal from a resistive sensor into a digital signal with the goal to estimate the respiratory rate of a subject. To detect breathing, a known method based on a nasal thermistor, which detects temperature changes near the nostrils, is used. In this work, the thermistor mounted in a Wheatstone bridge, forms a $R C$ circuit which is connected directly to a microcontroller, without using any analog circuit or analog-digital converter. Thus, whenever the subject breathes, it causes a fractional change in resistance $x\left(\Delta R / R_{0}\right)$ on the thermistor, and this produces a time-modulated signal that is directly digitized with the microcontroller. Measurements were made on 23 volunteers, obtaining changes of $x>0.01$. The temperature resolution was $0.2{ }^{\circ} \mathrm{C}$, and the time response was $0.8 \mathrm{~s}$, mainly limited by the thermistor properties; these features were enough to obtain a well-defined waveform of the breathing, from which was easy to estimate the respiratory rate by a compact, low cost and low power consumption system. Unlike interface circuits based on voltage or current amplitude, with this kind of interface, the self-heating of the sensor is avoided since the thermistor does not require any voltage or bias current.

Keywords: time-based measurements, Wheatstone bridge sensors, respiratory rate, temperature measurement, nasal thermistor.

\section{Correspondencia:}

Rafael González Landaeta

Instituto de Ingeniería y Tecnología, Departamento de Ingeniería Eléctrica y Computación, Universidad Autónoma de Ciudad Juárez, Av. del Charro 450 nte., C.P.32310, Ciudad Juárez, Chihuahua, México.

Correo electrónico: rafael.gonzalez@uacj.mx
Fecha de recepción: 16 de diciembre de 2015

Fecha de aceptación: 7 de marzo de 2016 


\section{RESUMEN}

Este trabajo propone un sistema que convierte una señal modulada en tiempo, proveniente de un sensor resistivo, en una señal digital con el fin de estimar la frecuencia respiratoria de un sujeto. Para detectar la respiración se utilizó el método basado en un termistor nasal, el cual detecta los cambios de temperatura cerca de las fosas nasales. En este trabajo, el termistor, montado en un puente de Wheatstone, forma un circuito $R C$ que se conecta directamente a un microcontrolador, sin necesidad de usar ningún circuito analógico, ni conversor analógico-digital. Así, cada vez que el sujeto respire, provoca un cambio fraccional de resistencia $x\left(\Delta R / R_{0}\right)$ en el termistor, y esto produce una señal modulada en tiempo que se digitaliza directamente con el microcontrolador. Se hicieron medidas en 23 voluntarios, obteniendo cambios de $x>0.01$. Se obtuvo una resolución en temperatura de $0.2{ }^{\circ} \mathrm{C}$ y un tiempo de respuesta de $0.8 \mathrm{~s}$, limitado principalmente por las propiedades del termistor utilizado. Estas características demostraron ser suficientes para obtener una forma de onda de la respiración bien definida, de la cual es sencillo estimar la frecuencia respiratoria mediante un sistema compacto, de bajo costo y bajo consumo de energía. A diferencia de los circuitos de interfaz basado en la amplitud de tensión o corriente, con este tipo de interfaz se evita el autocalentamiento del sensor, ya que el termistor no requiere ningún voltaje o corriente de polarización.

Palabras clave: medidas basadas en el tiempo, sensores en puente de Wheatstone, frecuencia respiratoria, medida de temperatura, termistor nasal.

\section{INTRODUCTION}

Respiratory rate (RR) is one of the basic physiological parameters that can help to assess the health condition of a subject. Several works have proposed different methods for detecting the breathing waveform and have estimated parameters of interest related to the ventilation of the subject [1-3]. Nasal thermistor is a wellknown and accurate method for recording the respiratory phase, which reacts to variations in air temperature [4]. Formerly, this method was used for assessing respiratory patterns and nocturnal events in studies aimed to diagnose sleep disorders [5]. Nowadays, it is still a reference in several polysomnographic studies in the diagnosis of obstructive sleep apnea $[6,7]$.

Resistive sensors, as thermistors, conveniently are set in voltage dividers or in a Wheatstone bridge, which are suited for sensors with large resistance variations and nonlinear response [8]. With an appropriate configuration, it is possible to linearize the response of such sensors with circuits that provides an output voltage (or current) that depends on both the resistance variations of the sensor and the supply voltage (or current). In these conventional circuits, it is necessary some analog signal processing stages to adapt the voltage range to that of the analog-to-digital converter (ADC). Furthermore, the polarization stage can cause a self-heating problem, increasing the uncertainty of the measurement process. To enhance resolution, it has been proposed synchronous demodulation, which adds extra processing stages on the measurement system [9].

This paper proposes a novel system based on a direct sensor-to-microcontroller $(\mu \mathrm{C})$ interface circuit (time-modulated circuit). The system is implemented by a thermistor in a Wheatstone quarter-bridge topology directly connected to a $\mu \mathrm{C}$ without any analog processing circuits, nor ADC; which results in a compact solution to 
detect temperature variations. Such a direct interface circuit relies on measuring the discharging time of a $R C$ network that includes the resistances of the sensor bridge, and by means of a time-based equation, it is possible to estimate the fractional resistance change $x\left(\Delta R / R_{0}\right)$ of the sensor [10].

In order to demonstrate the feasibility of the proposed method, we used the wellknown nasal thermistor technique to detect the breathing by measuring the thermal fluctuations near the nostrils of several subjects. Although the proposed method has been successfully applied to piezoresistive and magnetoresistive sensors in full and half-bridge topologies $[11,12]$ to detect DC or quasi-DC signals, at the best of our knowledge, such method has not been used to sense AC magnitudes (such as respiratory rate), which could be extended to other medical applications.

\section{SENSING APPROACH}

\section{Thermistor description}

In order to detect the temperature variations near the nostrils, it is necessary to know the thermistor characteristics (e.g., sensitivity and time response). Commonly, a normal breathing of an adult subject is between 12 and 15 breaths per minute, that is, a bandwidth up of $0.25 \mathrm{~Hz}$ [13]. Temperature fluctuations $(\Delta T)$ due to the subject breath depend on the environmental temperature, and it rarely exceeds $20{ }^{\circ} \mathrm{C}$ if the surrounding temperature is $13{ }^{\circ} \mathrm{C}[14,15]$. In order to obtain a breathing waveform to estimate the $\mathrm{RR}$, we considered that the system must be able, in principle, to have a resolution of $0.5^{\circ} \mathrm{C}$.

The thermistor used in this study is the NTCLE305E4202SB (VISHAY). It has a negative temperature coefficient (NTC), and the resistance $R_{T}$ at any temperature $T$ (over a $50{ }^{\circ} \mathrm{C}$ span) can be determined by an exponential law [8]:

$$
R_{T}=R_{0} e^{B\left(\frac{1}{T}-\frac{1}{T_{0}}\right)},
$$

where $B$ is the characteristic temperature of the material, $R_{0}$ is the resistance of the thermistor at a reference temperature $T_{0}$, usually $25{ }^{\circ} \mathrm{C}$. Here, we considered a temperature span of $25{ }^{\circ} \mathrm{C} \quad\left(15{ }^{\circ} \mathrm{C}\right.$ $40{ }^{\circ} \mathrm{C}$ ) where the relative sensitivity of the thermistor $(\alpha)$ has a nonlinear dependence on $T$ :

$$
\alpha=-\frac{B}{T^{2}}
$$

From (2), $\alpha_{15}=-4.23 \% / \mathrm{K}, \alpha_{25}=-3.95$ $\% / \mathrm{K} \alpha_{40}=-3.58 \% / \mathrm{K}$, which corresponds to a maximal relative non-linearity error of 15 $\%$ (calculated from the best-fit straight line). Theoretically, for a temperature resolution of $0.5{ }^{\circ} \mathrm{C}$, the fractional resistance change of the thermistor must be $2 \%(x=0.02)$, which implies an effective resolution of 6 bit. According to [10], the method used to measure $x$ has an effective resolution of 8 bit for a measuring time of $10 \mathrm{~ms}$, and it has a theoretical resolution of $0.1{ }^{\circ} \mathrm{C}$. Nonlinear errors modify the shape of the temperature variations, but not the estimation of the $R R$ [16]. A thermistor behaves as low-pass filter, and the bandwidth depends on the thermal constant $\tau_{s}$. Thus, a high value in $\tau_{s}$ can produce a time delay in the estimation of the RR. Commonly, $\tau_{s}$ is often provided by the manufacturer, but under specific conditions; nevertheless, we can estimate $\tau_{s}$ from a simple experimental setup in order to assert this value. Table 1 shows the basic characteristics given by the manufacturer of the thermistor used.

\section{Fundamentals of the interface circuit}

Wheatstone bridges with resistive sensors (quarter-bridge, half-bridge, and full-bridge) can be directly connected to a $\mu \mathrm{C}$ by using time-based measurement circuits that yield a digital output that is proportional to the change of $x$. 
Table 1: Basics characteristic of the thermistor NTCLE305E4202SB.

\begin{tabular}{lcc}
\hline \multicolumn{1}{c}{ Parameter } & Value & Unit \\
\hline Resistance value & 2060 & $\Omega$ \\
at $25{ }^{\circ} \mathrm{C}\left(R_{0}\right)$ & & \\
Tolerance on $R_{25-}$ value & \pm 1.92 & $\%$ \\
$B$ & 3511 & $\mathrm{~K}$ \\
Operating Range & -40 to +125 & ${ }^{\circ} \mathrm{C}$ \\
Response time & & \\
(in stirred air) & $\approx 3$ & $\mathrm{~s}$ \\
(in oil) & $\approx 0.7$ & \\
Weight & $\approx 0.05$ & $\mathrm{~g}$ \\
\hline
\end{tabular}

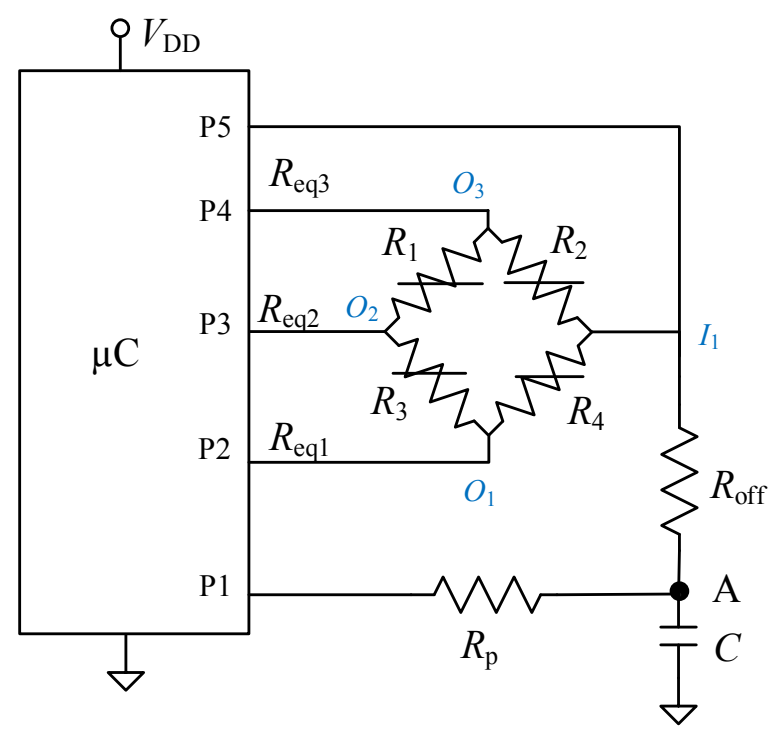

Figure 1: Direct sensor-to- $\mu \mathrm{C}$ interface circuit for resistive bridge sensors.

Figure 1 shows a direct interface circuit for resistive bridge sensors, previously analyzed in $[10,17]$. In this kind of interface, the resistive bridge is considered a network with one input terminal and three output terminals. To estimate the changes on $x$, the direct interface circuit performs four discharging times measurements $\left(t_{\mathrm{d} 1}, t_{\mathrm{d} 2}, t_{\mathrm{d} 3}\right.$ and $\left.t_{\mathrm{d} 4}\right)$ and applies a time-based equation accordingly with the bridge topology [10].

The measurement of each discharging time, $t_{\mathrm{d}}$, involves two stages: (a) charging and (b) discharging and time measurement. First, $C$ is charged through $R_{\mathrm{p}}$ (at least $5 R_{\mathrm{p}} C$ ) towards $V_{\mathrm{DD}}$. Then $C$ is discharged towards $V_{\mathrm{SS}}$ (ground reference) through each equivalent resistance, $R_{\text {eqi }}$, between node $\mathrm{A}$ and pins $\mathrm{P} 2-\mathrm{P} 5$, respectively, which results in a $R C$ circuit with a time constant $\tau=$ $R_{\text {eq } i} C$. During the discharging time, when the voltage across $C$ reaches $V_{\mathrm{TL}}$ (low threshold voltage of the Schmitt Trigger (ST) input) on pin $\mathrm{P} 1$, the timing process stops. The count of the embedded timer is the digital equivalent to the discharging time $t_{\mathrm{d}}$. Figure 2 shows the voltage waveform across $C$ during the measurement, which is accomplished in eight steps. Table 2 summarizes the $\mu \mathrm{C}$ pins configuration during the measurement sequence.

A quarter-bridge topology is considered when $R_{1}=R_{2}=R_{3}=R_{0}$ and $R_{4}=R_{0}(1-$ $x)$, such a NTC thermistor (Figure 1). In this case, the respective equivalent resistances seen from pins P2-P5 and node A (when the internal resistance $R_{\text {in } i}$ of each $\mu C$ pin are considered) are:

$$
\begin{aligned}
& R_{\mathrm{eq} 1}=\frac{R_{0}(3-3 x)}{4-x}+R_{\mathrm{off}}+R_{\mathrm{in} 2} \\
& R_{\mathrm{eq} 2}=\frac{R_{0}(4-2 x)}{4-x}+R_{\mathrm{off}}+R_{\mathrm{in} 3} \\
& R_{\mathrm{eq} 3}=\frac{R_{0}(3-x)}{4-x}+R_{\mathrm{off}}+R_{\mathrm{in} 4} \\
& R_{\mathrm{eq} 4}=R_{\mathrm{off}}+R_{\mathrm{in} 5}
\end{aligned}
$$

In such conditions, the respective discharging time through each equivalent resistance is:

$$
t_{\mathrm{di}}=R_{\mathrm{eq} i} C \ln \left(\frac{V_{\mathrm{DD}}}{V_{\mathrm{TL}}}\right)
$$

The time-based equation, originally proposed in [10] and improved in [17], to estimate $x$ in a quarter-bridge topology is:

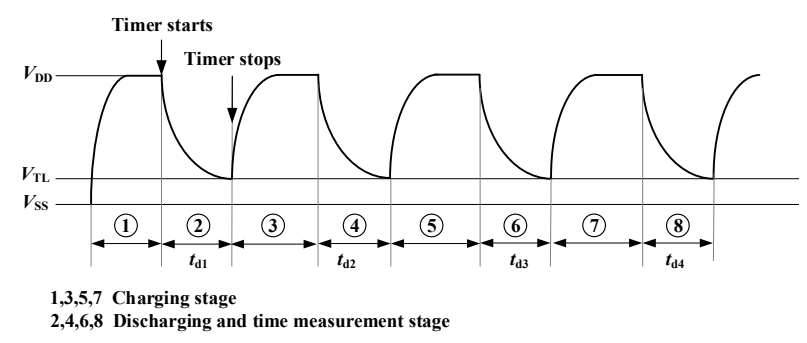

Figure 2: Voltage waveform across $C$ during a full measurement sequence. 
Table 2: Configuration of the $\mu \mathrm{C}$ pins during the measurement sequence.

\begin{tabular}{cccccc}
\hline Step & P1 & P2 & P3 & P4 & P5 \\
\hline 1,3 & Output "1" & Input & Input & Input & Input \\
5,7 & & & & & \\
2 & Capture & Output "0" & Input & Input & Input \\
4 & Capture & Input & Output "0" & Input & Input \\
6 & Capture & Input & Input & Output "0" & Input \\
8 & Capture & Input & Input & Input & Output "0" \\
\hline
\end{tabular}

$$
x^{*}=\frac{2\left(t_{\mathrm{d} 1}-t_{\mathrm{d} 3}\right)}{t_{\mathrm{d} 2}+t_{\mathrm{d} 3}-t_{\mathrm{d} 1}-t_{\mathrm{d} 4}}
$$

Replacing (3) in (4), and subsequently in (5), yields:

$$
\begin{aligned}
x^{*}= & \frac{R_{0}}{R_{0}+\Delta R_{\text {in } 35}+\Delta R_{\text {in } 42}} x \\
& +\frac{2 \Delta R_{\text {in } 24}}{R_{0}+\Delta R_{\text {in } 35}+\Delta R_{\text {in } 42}}
\end{aligned}
$$

Gain and offset errors are small because they depend on the differences between $R_{\operatorname{in} i}$ of the $\mu \mathrm{C}$. For instance, if the internal resistances are equals, the errors will be zero. On the other hand, those errors can be corrected by calibration. The resistance $R_{\mathrm{p}}$ in Figure 1 is included to improve the rejection of power supply interferences in the charging stage $[18,19] . R_{\text {off }}$ was included to reduce the effects of $R_{\text {ini }}$ [17], also this resistance limits the maximal current sunk by each pin during the discharging and time measurement. In $[18,19]$, there are some design guidelines to improve the performance of the direct interface circuits and therefore the measurement.

\section{DESIGN AND IMPLEMENTATION}

\section{Design of the measurement system}

Figure 3 shows the proposed circuit for detecting the breathing. It was implemented by the microcontroller MSP430F123 (Texas Instruments) running at $8 \mathrm{MHz}$ (quartz oscillator clock). So, the embedded 16-bit timer/counter counts the discharge time by incrementing its value each 125 ns. The supply voltage of the $\mu \mathrm{C}$ was $V_{\mathrm{DD}}=3.0 \mathrm{~V}$, provided by a dedicated voltage regulator (LF30CV) to reduce power supply interference, which may result in trigger noise [19]. The function of P1-P5 (Figure 1) was implemented by P1.2, P3.7, P3.6, P3.3, and P3.2, respectively. A quarter-bridge topology was implemented by $R_{1}=R_{2}=R_{3}=R_{0}=$ $2.2 \mathrm{k} \Omega$ resistances (with $1 \%$ of tolerance and $50 \mathrm{ppm}$ ). The resistance of the NTC at 25 ${ }^{\circ} \mathrm{C}$ was close to $2.06 \mathrm{k} \Omega$ (see Table 1 ). The thermistor was placed on $R_{4}=R_{0}(1-x)$.

To reduce the effects of the internal trigger noise, the $\mu \mathrm{C}$ was set in LPM2 mode. This option disables the CPU but remains in active mode timers and interrupts, while the discharging times are being measured as suggested in [19]. The pin P1.2 (external interrupt with ST buffer, capture mode) was configured to interrupt the $\mu \mathrm{C}$ on falling edge every time the discharging $C$ voltage reaches the $V_{\mathrm{TL}}$ value. The $\mu \mathrm{C}$ program was written in $\mathrm{C}$ language; however, to increase precision in time measurements, the sequences shown in Table 2 were written in assembler language. $C$ was selected to obtain a suitable time constant, $\tau=R_{\text {eq } i} C$, for the discharging and time measurement stage. A large $\tau$ value implies a slow slew rate of the exponential voltage waveform at the trigger point, which makes the triggering process more susceptible to noise, increasing the count dispersion and the standard deviation of the measurement. In contrast, a too small value of $\tau$ yields few counts, giving a large quantization error. Thus, the optimal time constant value was experimentally determined, and it was between 2 and $3 \mathrm{~ms}$ [10, 19]. Therefore, we selected $C=1 \mu \mathrm{F}$, with $\pm 5 \%$ of tolerance and $100 \mathrm{ppm} /{ }^{\circ} \mathrm{C}$ of temperature coefficient. We chose $R_{\mathrm{p}}=100 \Omega$ that results in charging times $\left(5 R_{\mathrm{p}} C\right)$ of $500 \mu \mathrm{s}$.

The discharging times $t_{\mathrm{d} 1}, t_{\mathrm{d} 2}, t_{\mathrm{d} 3}$ and $t_{\mathrm{d} 4}$ were measured, and $x$ was estimated by (5). Then, this value was sent to a PC via RS-232 by a control program in LabVIEW ${ }^{\mathrm{TM}}$. The serial communication interface was implemented by a MAX3223 supplied by a separated voltage regulator (and was set in shutdown mode during 


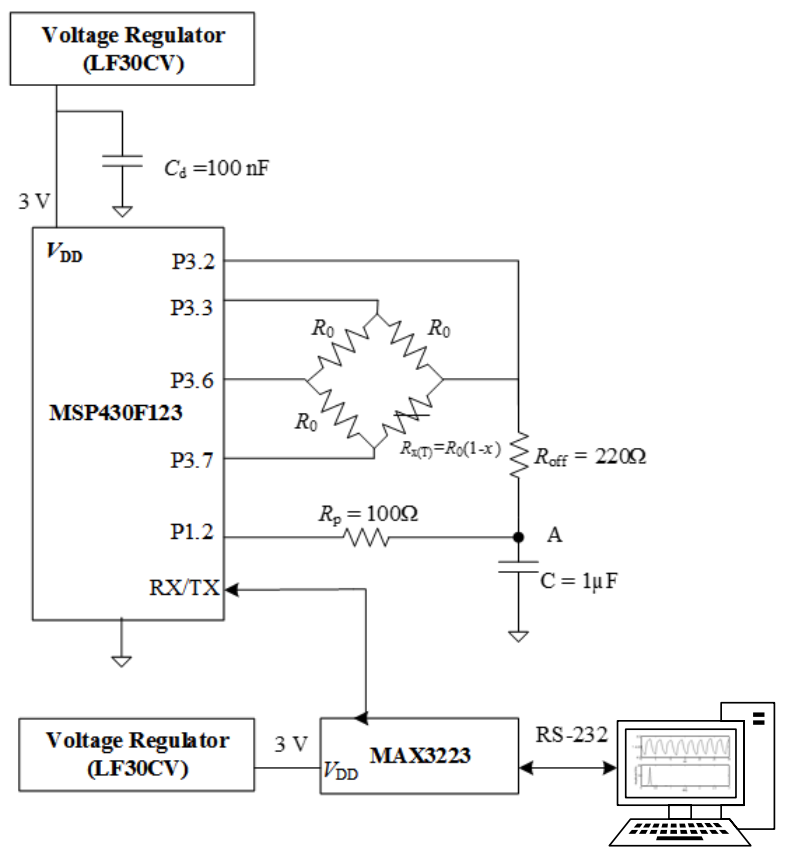

Figure 3. Time-based measurement system for detecting respiratory rate.

the measuring process) to prevent induced transients in the power supply that could affect the discharging process [19].

\section{Measurement protocol}

The process to validate the proposed method was done over 23 volunteers ( 8 women and 15 men), all with distinct physical characteristics: (mean \pm SD: age $=(27 \pm$ 8) years; weight $=(77 \pm 15) \mathrm{kg}$; height $(1.72 \pm 0.09) \mathrm{m}$. We measured thermal fluctuations by placing the thermistor near to the nostrils of each subject. As a reference signal, a piezoelectric sensor LDT1028K from Measurement Specialties [20], was attached to the chest of each volunteer in order to detect the movements of the thorax on each breathing. Figure 4 depicts the location of each sensor on the subject (a) and the position of the thermistor near the nostrils (b). The signal of the piezoelectric sensor was processed by a charge amplifier with a sensitivity of $-212 \mathrm{mV} / \mathrm{pC}$ and filtered by a first-order low-pass filter with a corner frequency of $1 \mathrm{~Hz}$.

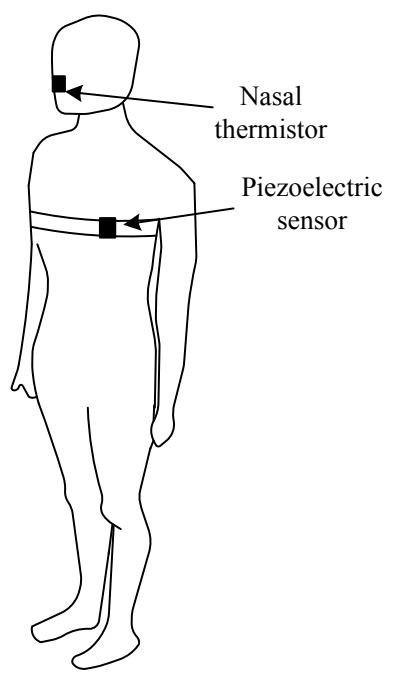

a)

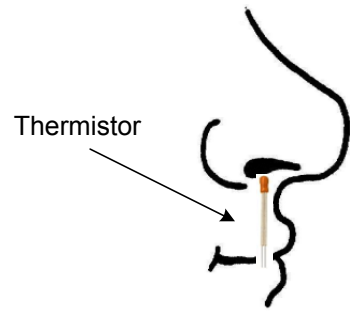

b)
Figure 4. (a) Location of the sensors on the body during the measurement protocol and (b) position of the thermistor near the nostrils.

The measurements were obtained by two procedures. In the first procedure called "Controlled Breathing", every subject was asked to breathe following a baseline of an oscilloscope that showed a sinusoidal signal with $1 \mathrm{~V}$ peak-to-peak and $0.25 \mathrm{~Hz}$. In the second procedure called "Free Breathing", the tests were performed while the subjects were breathing at their own rhythm. On each subject, the test was repeated three times with a measurement time of $30 \mathrm{~s}$ each test. The signal obtained from nasal thermistor was compared with that obtained from a piezoelectric sensor.

\section{RESULTS AND DISCUSSION}

In the proposed system, the low-pass response is limited by the time response of the thermistor. Figure 5 depicts the fractional resistance of the thermistor as a response to a thermal step input, which was between 23 ${ }^{\circ} \mathrm{C}$ and $9{ }^{\circ} \mathrm{C}$. We obtained $\tau_{s}=0.8 \mathrm{~s}$, which is suitable for detecting the RR. The nonlinear errors of the thermistor were not considered because we were only interested on the detection of an $\mathrm{AC}$ magnitude (temperature fluctuations). 


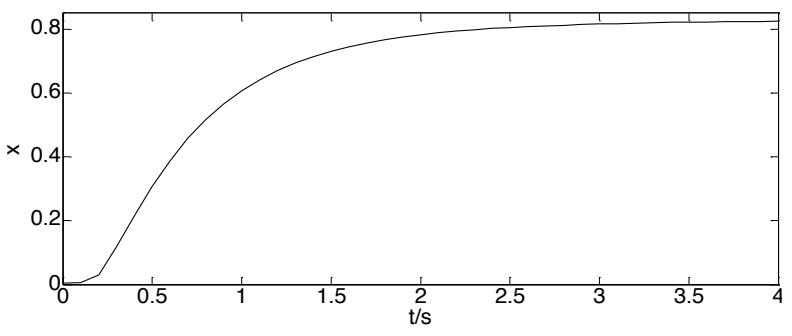

Figure 5. Experimental time response of the thermistor NTCLE305E4202SB for a thermal step between $23{ }^{\circ} \mathrm{C}$ and $9{ }^{\circ} \mathrm{C}$. The sensor is able to respond in $0.8 \mathrm{~s}$, enough for detecting the breathing-related thermal fluctuations.
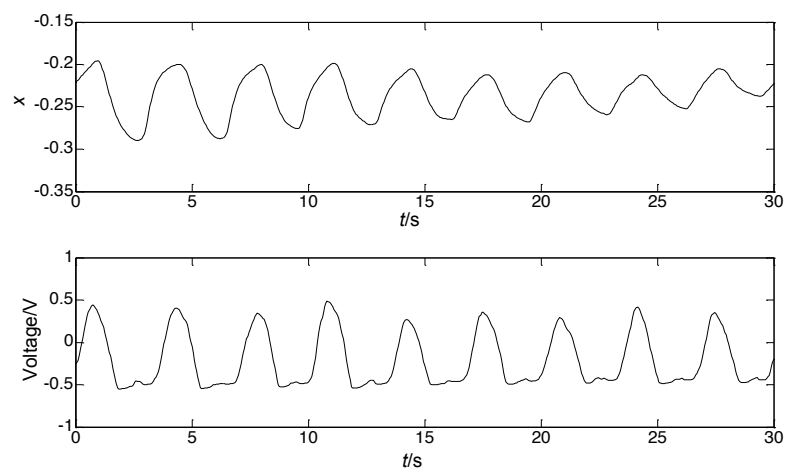

Figure 6. Controlled Breathing waveform obtained from a nasal thermistor directly connected to a $\mu \mathrm{C}$ (top) and from a piezoelectric film sensor attached to the chest of a volunteer (bottom).

Figure 6 shows the signals obtained with the proposed system and with the piezoelectric sensor. Both signals match in the number of breaths and also coincide with the $0.25 \mathrm{~Hz}$ of the Controlled Breathing measured during the $30 \mathrm{~s}$.

Figures 7 and 8 show the frequency spectrum of the breathing waveforms of two subjects who were asked to breathe at their own rhythm. The signals were obtained with the direct interface circuit. As can be seen, both figures presented remarkable differences. For example, Figure 7 shows a clear peak at $0.3 \mathrm{~Hz}$ (18 breaths per minute) while Figure 8 shows a clear peak at $0.16 \mathrm{~Hz}$ (10 breath per minute). These results demonstrate that the proposed system is able to detect $R R$ breath-by-breath, and it is also able to detect different breathing rates. Moreover, the frequency spectrum of both signals shows a negligible contribution of noise.
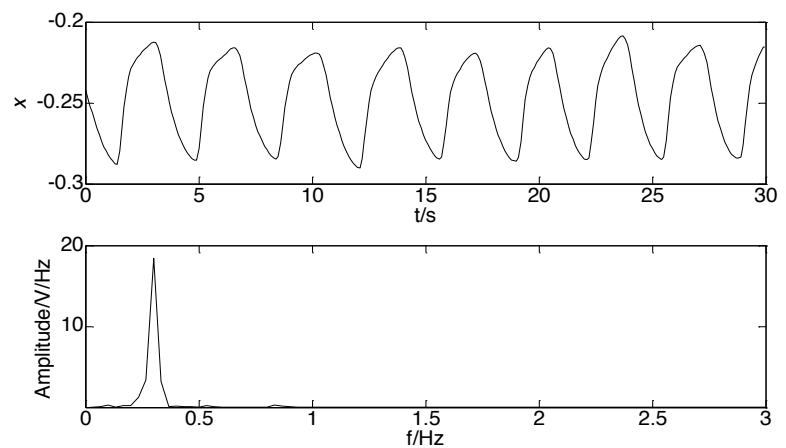

Figure 7. Free Breathing waveform obtained from a nasal thermistor directly connected to a $\mu \mathrm{C}$ (top) and the frequency spectrum showing a clear peak at 0.3 $\mathrm{Hz}$.
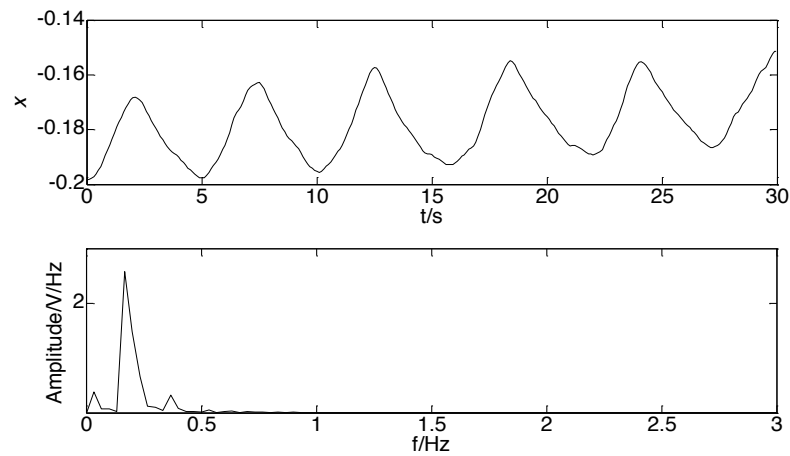

Figure 8. Free Breathing waveform obtained from a nasal thermistor directly connected to a $\mu \mathrm{C}$ (top) and the frequency spectrum showing a clear peak at 0.16 $\mathrm{Hz}$.

The sensitivity of the proposed method relies on the sensor sensitivity. Since the thermistor is not supplied by any constant voltage or current, self-heating problems are avoided and the sensitivity does not depends on any polarization source, as usually happens in conventional signal conditioning systems.

Figure 9 shows a Bland-Altman plot that compares the $\mathrm{RR}$ time interval of the breathing signals, detected from the 23 volunteers with the proposed method and with the piezoelectric sensor. The mean bias was $17 \mathrm{~ms}$ and the dispersion (with a $95 \%$ confidence interval) was about $303 \mathrm{~ms}$, which is practically negligible. Figure 10 shows the scatter plot and the correlation coefficient for the RR time interval, where both were estimated from signals detected with the 


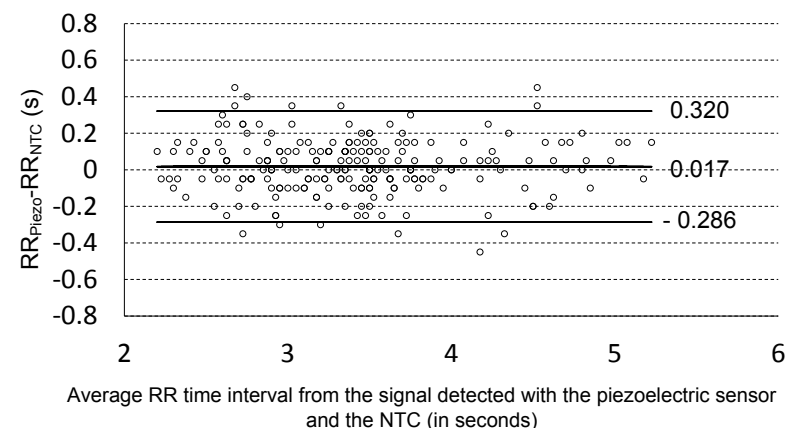

Figure 9. Bland-Altman plot of each RR time interval detected from the signal obtained with the thermistor and with the piezoelectric sensor of the 23 volunteers.

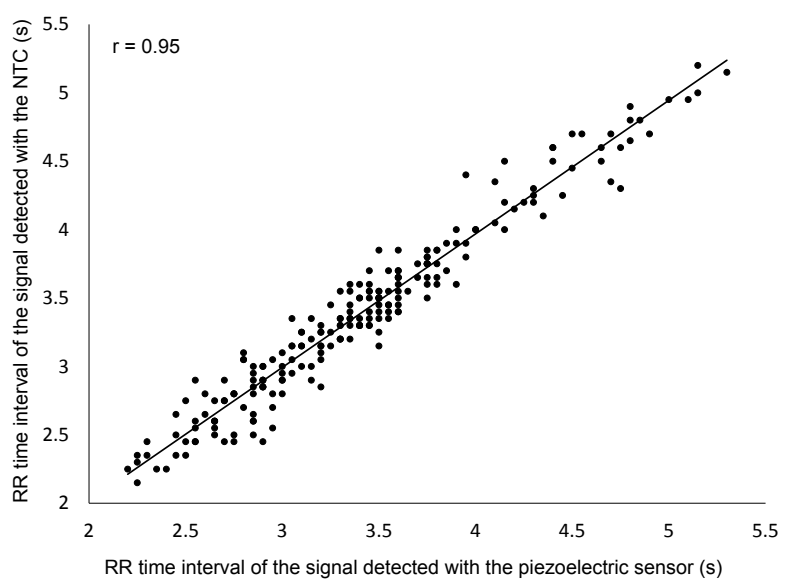

Figure 10. Correlation analysis of the RR time interval estimated from the signal detected with the thermistor and the RR time interval estimated from the signal detected with the piezoelectric sensor.

proposed method and with the piezoelectric sensor. The correlation coefficient for the data on the 23 volunteers was 0.95 , which is a statistically significant correlation.

\section{CONCLUSIONS}

A simple, low-cost and compact system has been implemented for detecting the breathing using a nasal thermistor. The sensor, mounted in a Wheatstone quarter-bridge topology, was directly connected to a $\mu \mathrm{C}$. The temperature fluctuations near the nostrils (due to breathing of the subjects) have been clearly detected. The proposed system did not require the use of any analog processing circuits, nor ADC. The proposed circuit could detect fractional changes in resistance of $x>0.01$, which resulted in a resolution of $0.2{ }^{\circ} \mathrm{C}$, enough to follow the breathing-related temperature fluctuations. This achieved well-shaped breathing waveforms, with a negligible noise contribution, from which it is easy to estimate the RR. The system was able to detect the respiration breath-by-breath, just by measuring the discharging time in a capacitor. It was not necessary to supply any voltage or current to the thermistor, so the self-heating was avoided. The methodology proposed in this research can be considered in other medical applications, where the temperature measurement (e.g., the body temperature) could be obtained by a resistive sensor. Bland-Altman and Scatter plots were used to compare de RR time interval between the signal detected with the thermistor and that detected with the piezoelectric sensor. The calculated mean bias was less than 17 ms, and the dispersion was lower than $303 \mathrm{~ms}$, and correlation coefficient was 0.95 , which is statistically significant.

\section{ACKNOWLEDGMENTS}

This work has been funded by PRODEP (Programa para el Desarrollo Profesional Docente) and UACJ (Universidad Autónoma de Ciudad Juárez) México, project UACJPTC-327.

\section{REFERENCES}

1. Schäfer A., et al., "Estimation of breathing rate from respiratory sinus arrhythmia: comparison of various methods," Ann. of Biomed. Eng., vol. 36, no 3, pp. 476-485, Jan. 2008.

2. Karlen W., et al., "Multiparameter respiratory rate estimation from the photoplethysmogram," IEEE Trans. on Biomed. Eng., vol. 60, no 7, pp. 19461953, Feb. 2013.

3. Chan A. M., et al., "Ambulatory respiratory rate detection using ECG 
and triaxial accelerometer," in 35th Conf. of the IEEE Eng. in Med. and Biol. Soc., Osaka, 2013, pp. 4058-4061.

4. Carskadon M. A., et al., "Respiration during sleep in children," Western Journal of Medicine, vol. 128, pp. 477481, Jun. 1978.

5. American Thoracic Socienty "Standards and indications for cardiopulmonary sleep studies in children," Am. J Respir. Crit. Care Med., vol. 153, no. 2, pp. 866878, Feb. 1996.

6. Dinç A. E., et al, "Reliability of Sleep Strip as a screening test in obstructive sleep apnea patients," Eur. Arch. of Otorhinolaryngol., vol. 271, no. 10, pp. 1-6, Oct. 2014.

7. Tan H. L., et al., "Overnight Polysomnography versus Respiratory Polygraphy in the Diagnosis of Pediatric Obstructive Sleep Apnea," Sleep, Vol. 37, no. 2, pp. 255-260, Feb. 2014.

8. Pallàs-Areny R., Webster J. G., "Sensors and Signal Conditioning 2nd Edition", John Wiley \& Sons, New York, 2001, pp. 94-109.

9. Cuadras A., Casas O., "Determination of heart rate using a high-resolution temperature measurement," IEEE Sensors J., Vol. 6, no. 3, pp. 836-843, Jun. 2006 .

10. Sifuentes E., et al., "Direct interface circuit to linearise resistive sensor bridges," Sensors and Actuators A, vol. 147, no. 1, pp. 210-215, Sep. 2008.

11. Jordana J., Pallàs-Areny R., "A simple, efficient interface circuit for piezoresistive pressure sensors," Sensors and Actuators A, vol 127, no. 1, pp. 6973, Feb. 2006.
12. Sifuentes E., et al., "Wireless magnetic sensor node for vehicle detection with optical wake-up," IEEE Sensors J., vol. 11, no. 8, pp. 1669-1676, Jan. 2011.

13. Brown B. H., et al., "Medical Physics and Biomedical Engineering," Institute of Physics, Madison, 2001, pp. 548-577.

14. Toba E., et al., "Non-Invasive Measurement System for Human Respiratory Condition and Body Temperature," in IEEE Int. Conf. on MFI, Las Vegas NV, 1994, pp. 770-783.

15. Storck K., et al., "Heat Transfer Evaluation of the Nasal Thermistor Technique," IEEE Trans. On Biomed. Eng., vol. 43, no. 12, pp. 1187-1191, Dec. 1996.

16. Farre R., et al., "Accuracy of thermistors and thermocouples as flow-measuring devices for detecting hypopnoeas," Eur. Respir. J., vol. 11, no.1, pp. 179-182, Jan. 1998.

17. Sifuentes E., et al., "Improved direct interface circuit for resistive full-and half-bridge sensors," in IEEE, 4th Int. Conf. on Electrical and Electronics Engineering, ICEEE 200\%, Mexico, 2007, pp. 197-200.

18. Reverter F., "The art of directly interfacing sensors to microcontrollers," Low Power Electronics and Applications J., vol 2, no. 4, pp 265-281, 2012.

19. Reverter F., "Direct interface circuits for sensors", in: S. Nihtianov and A. Luque, "Smart Sensors and MEMS: Intelligent Devices and Microsystems for Industrial Applications", Woodhead Publishing, Chap 2, pp 27-62, 2014.

20. LDT1-028K Shielded Piezo Sensors, Measurement Specialties, Hampton, VA, 2009, pp. 1-3. 
Revista Mexicana de Ingeniería Biomédica · volumen 37 · número 2 · May-Ago, 2016 thb 